\title{
Genome-wide identification and expression profile under abiotic stress of the barley non-specific lipid transfer protein gene family and its Qingke Orthologues
}

\author{
Jiecuo Duo ${ }^{1,2+}{ }^{1}$, Huiyan Xiong ${ }^{3 \dagger}$, Xiongxiong $\mathrm{Wu}^{1}$, Yuan $\mathrm{Li}^{1}$, Jianping $\mathrm{Si}^{1}$, Chao Zhang ${ }^{1}$ and Ruijun Duan ${ }^{1 *}$
}

\begin{abstract}
Background: Plant non-specific lipid transfer proteins (nsLTPs), a group of small, basic ubiquitous proteins to participate in lipid transfer, cuticle formation and stress response, are involved in the regulation of plant growth and development. To date, although the nsLTP gene family of barley (Hordeum vulgare L.) has been preliminarily identified, it is still unclear in the recently completed genome database of barley and Qingke, and its transcriptional profiling under abiotic stress has not been elucidated as well.

Results: We identified 40 barley nsLTP (HVLTP) genes through a strict screening strategy based on the latest barley genome and 35 Qingke nsLTP (HtLTP) orthologues using blastp, and these LTP genes were divided into four types $(1,2, D$ and $G)$. At the same time, a comprehensive analysis of the physical and chemical characteristics, homology alignment, conserved motifs, gene structure and evolution of HVLTPs and HtLTPs further supported their similar nsLTP characteristics and classification. The genomic location of HVLTPS and HtLTPs showed that these genes were unevenly distributed, and obvious HVLTP and HtLTP gene clusters were found on the 7 chromosomes including six pairs of tandem repeats and one pair of segment repeats in the barley genome, indicating that these genes may be co-evolutionary and co-regulated. A spatial expression analysis showed that most HVLTPs and HtLTPS had different tissue-specific expression patterns. Moreover, the upstream cis-element analysis of HVLTPS and HtLTPS showed that there were many different stress-related transcriptional regulatory elements, and the expression pattern of HVLTPS and HtLTPS under abiotic stress also indicated that numerous HVLTP and HLLTP genes were related to the abiotic stress response. Taken together, these results may be due to the differences in promoters rather than by genes themselves resulting in different expression patterns under abiotic stress.
\end{abstract}

Conclusion: Due to a stringent screening and comprehensive analysis of the nsLTP gene family in barley and Qingke and its expression profile under abiotic stress, this study can be considered a useful source for the future studies of nSLTP genes in either barley or Qingke or for comparisons of different plant species.

Keywords: Barley, Qingke, Non-specific lipid transfer proteins, Gene family, Expression profile, Abiotic stress

\footnotetext{
*Correspondence: 1995990043@qhu.edu.cn

${ }^{\dagger}$ Jiecuo Duo and Huiyan Xiong contributed equally to this work.

${ }^{1}$ College of Eco-Environmental Engineering, Qinghai University, Xining

810016, Qinghai Province, China

Full list of author information is available at the end of the article
}

(c) The Author(s). 2021 Open Access This article is licensed under a Creative Commons Attribution 4.0 International License, which permits use, sharing, adaptation, distribution and reproduction in any medium or format, as long as you give appropriate credit to the original author(s) and the source, provide a link to the Creative Commons licence, and indicate if changes were made. The images or other third party material in this article are included in the article's Creative Commons licence, unless indicated otherwise in a credit line to the material. If material is not included in the article's Creative Commons licence and your intended use is not permitted by statutory regulation or exceeds the permitted use, you will need to obtain permission directly from the copyright holder. To view a copy of this licence, visit http://creativecommons.org/licenses/by/4.0/. The Creative Commons Public Domain Dedication waiver (http://creativecommons.org/publicdomain/zero/1.0/) applies to the data made available in this article, unless otherwise stated in a credit line to the data. 


\section{Key message}

- 40 barley nsLTP (HvLTP) and 35 Qingke Orthologues nsLTP (HtLTP) genes were identified through stringent screening using the new barley and Qingke genomes and divided into four types (1, 2, D and G) by comprehensive analysis.

- The HvLTPs and HtLTPs were unevenly distributed in barley and Qingke chromosomes and had obvious gene clusters.

- Combined with the analysis of upstream ciselements of HvLTPs and HtLTPs and their expression pattern under abiotic stress, it was found that numerous $H \nu L T P$ and HtLTP genes may change their regulatory modes due to different upstream cis-elements and cause different abiotic stress responses.

\section{Background}

Plant lipid transfer protein (LTPs), named for their function that transfer phospholipids and fatty acids between cell membranes in vitro, and they are also known as non-specific LTPs (nsLTPs) because of the characteristic of non-specific binding to different lipids $[1,2]$. Plant nsLTPs are usually 6.5 to $10.5 \mathrm{kDa}$, although some nsLTPs are approximately $15 \mathrm{kDa}$, and their isoelectric points (pIs) are usually between 8.5 and 12 (occasionally less than 5). Therefore, nsLTPs are a group of small, basic and ubiquitous proteins, that have eight cysteine residue motifs (8CM, ECM) in a highly conserved backbone sequence, $\mathrm{C}-\mathrm{Xn}-\mathrm{C}$ -

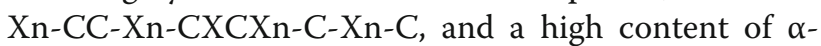
helices with a central hydrophobic cavity to bind lipids. Almost all nsLTPs carry an $\mathrm{N}$-terminal signal peptide in their nascent polypeptides [3-5]. Plant nsLTPs are involved in multiple physiological functions, such as cuticular lipid transport, cutin synthesis, cell wall extension, pollen development, pollen tube growth and guidance, stigma and pollen adhesion, plant signalling, and seed maturation $[1,6-8]$. In addition, some plant nsLTPs have been identified as related allergens in plant food and pollen [9-11]. The expression of some nsLTPs can be induced by biotic and abiotic stresses, including low or high temperature, drought, heavy metal exposure and disease [12-18]. In particular, many studies have shown that nsLTP genes are closely related to abiotic stress resistance in plant $[9,12,13,19,20]$.

Barley (Hordeum vulgare L.) is the fourth largest crop in the world after wheat, rice, and maize, and it is one of the oldest food and feed crop in the world (http:// faostat.fao.org). It is an important crop in China that has high economic and food value, especially on the Qinghai-Tibet Plateau. Tibetan hulless barley (highland barley), called "Qingke" in Chinese and "nas" in Tibetan, is the principal cereal cultivated on the Qinghai-Tibetan
Plateau for at least 3500 years. Due to its high soluble dietary $\beta$-glucan and arabinoxylan contents, it is beneficial to human health and has attracted considerable interest [21-23]. Karen Skriver cloned and analysed the gene structure of the LTP1 gene in barley [24]. The crystal structure of this gene, specifically in ligand binding preferences, was also studied [25]. Then, some other barley genes (LTP2, LTP3, and LTP4) have been cloned in succession [26]. Interestingly, the barley nsLTP gene (blt4) was also found in the cold stress response [27, 28]. The latest physical, genetic and functional sequence assemblies of the barley and Qingke genomes were completed in 2012 and 2016 [29, 30] and 2015, 2018, and $2020[22,31,32]$, respectively, and they provided an important reference for future crop breeding, improvement, gene function and evolution research.

Barley has strong drought resistance, low-temperature tolerance, and salt and alkali capacity, and its environmental adaptation mechanism is complex $[19,20]$. Qingke is mainly cultivated on the Tibetan Plateau ( $>4000 \mathrm{~m}$ above sea level), which has high UV-B radiation, low temperatures and low barometric pressure, and thus may therefore have greater extreme environmental adaptability than cultivated barley from other regions [21, 31]. Currently, the nsLTP genes and their multigene families have been studied in many plants, including Arabidopsis, carrot, rape (Brassica napus), broccoli (Brassica oleracea), tobacco (Nicotiana tabacum), sugar beet (Beta vulgaris), sesame (Sesamum indicum), tomato (Solanum lycopersicum), maize (Zea mays), sorghum (Sorghum vulgare), cotton (Gossypium hirsutum), rice (Oryza sativa) and wheat (Triticum aestivum) $[1,4$, 9, 13, 17, 33-39]. For barley, although 70 barley nsLTP genes have been identified in 2012 barley genome [40], there are still many deficiencies that need further comprehensive research by using the genome database of barley recently completed in 2016 . At the same time, the aim is to further understand the molecular mechanism underlying the stronger ability of Qingke to adapt to extreme environments than barley. Therefore, in this study, nsLTP gene family members were identified and a bioinformatics analysis of barley and Qingke was performed through strict screening based on the latest barley and Qingke genomes. Using qRT-PCR technology, the expression profile of this gene family under abiotic stress was also discussed. The results of this study lay a foundation for further studies on the biological and molecular functions of nsLTP genes in barley and Qingke.

\section{Results}

Identification, sequence analysis and classification of HvLTPs and HtLTPs

To identify the entire collection of putative nonredundant nsLTP genes in the barley genome, an 
accurate search workflow of nsLTP identification and data mining was performed (Fig. 1). Initially, three search methods were used to identify barley nsLTP family members. First, 109 amino acid sequences were obtained after conducting a BLASTP analysis of the IPK Barley BLAST Server using previously reported nsLTP protein sequences of Arabidopsis (79), maize (63), cabbage (63) and rice (77) as queries (Table S1), and the redundancy was checked. Second, a total of 265 proteins with the conserved Tryp alpha amyl domain (Pfam domain PF00234) were retrieved by the HMM search method. Third, the 39 relevant barley nsLTP genes were downloaded by keyword searches from the NCBI, IPK and Phytozome databases. Then, the above results were merged, and the redundant sequences were examined and removed. Finally, all the barley deduced nsLTP protein sequences were downloaded from the NCBI and IPK databases and the presence of LTP domain cl07890 was verified by BatchWeb CD-Search and Pfam validation using the domain PF00234. After that initial identification step, a total of 160 putative nsLTP protein sequences were identified. Then, each of the deduced protein sequences was manually assessed through the analysis of the cysteine residue motifs (8CM), and 107 proteins lacking the Cys residues were omitted from the remaining set. In addition, 11 proteins lacking Nterminal signal sequences (NSS) were also excluded by NSS prediction, and 8 proteins with C-terminal glycosylphosphatidylinositol (GPI) anchors remained by GPI anchor signal prediction. Subsequently, 2 proline-rich proteins were also excluded, and no proteins similar to At2S1- At2S4 and RATI were found. Finally, we identified 40 nsLTPs in the whole barley genome and named them HvLTPs, and 35 nsLTPs were also identified in Qingke genome by local BLAST analysis with HvLTPs and named them HtLTPs (Table 1). Furthermore, to better understand the characteristics of the HvLTP and HtLTP proteins, we analysed the theoretical isoelectric point (pI) and molecular weight (MW) for all putative HvLTP and HtLTP proteins and summarized them in Table 1. As shown in Table 1, considering the mature form of nsLTPs, the average length is 132 aa (91-200 aa), with a molecular mass ranging from 9206.81 to 19,981.15 Da. The average Mw of HvLTPs is $13,344 \mathrm{Da}$, and the average theoretical pI of HvLTPs is 8.07, while the average Mw of HtLTPs is $13,238 \mathrm{Da}$ and average theoretical pI of HtLTPs is 7.94, which demonstrates its small basic properties with subtle differences. We also analysed the instability index, aliphatic index and grand average of hydropathicity (GRAVY) of the HvLTPs and

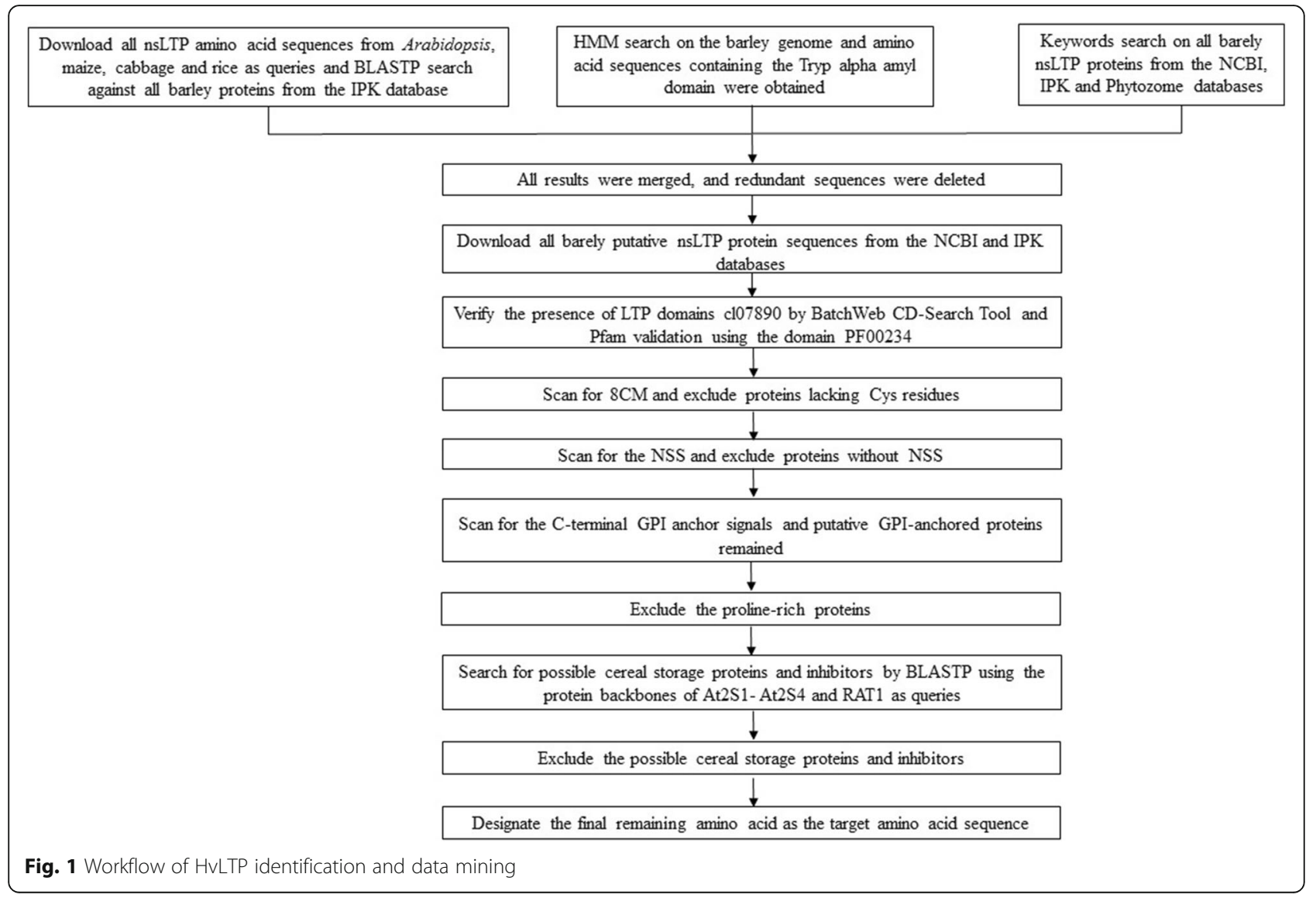




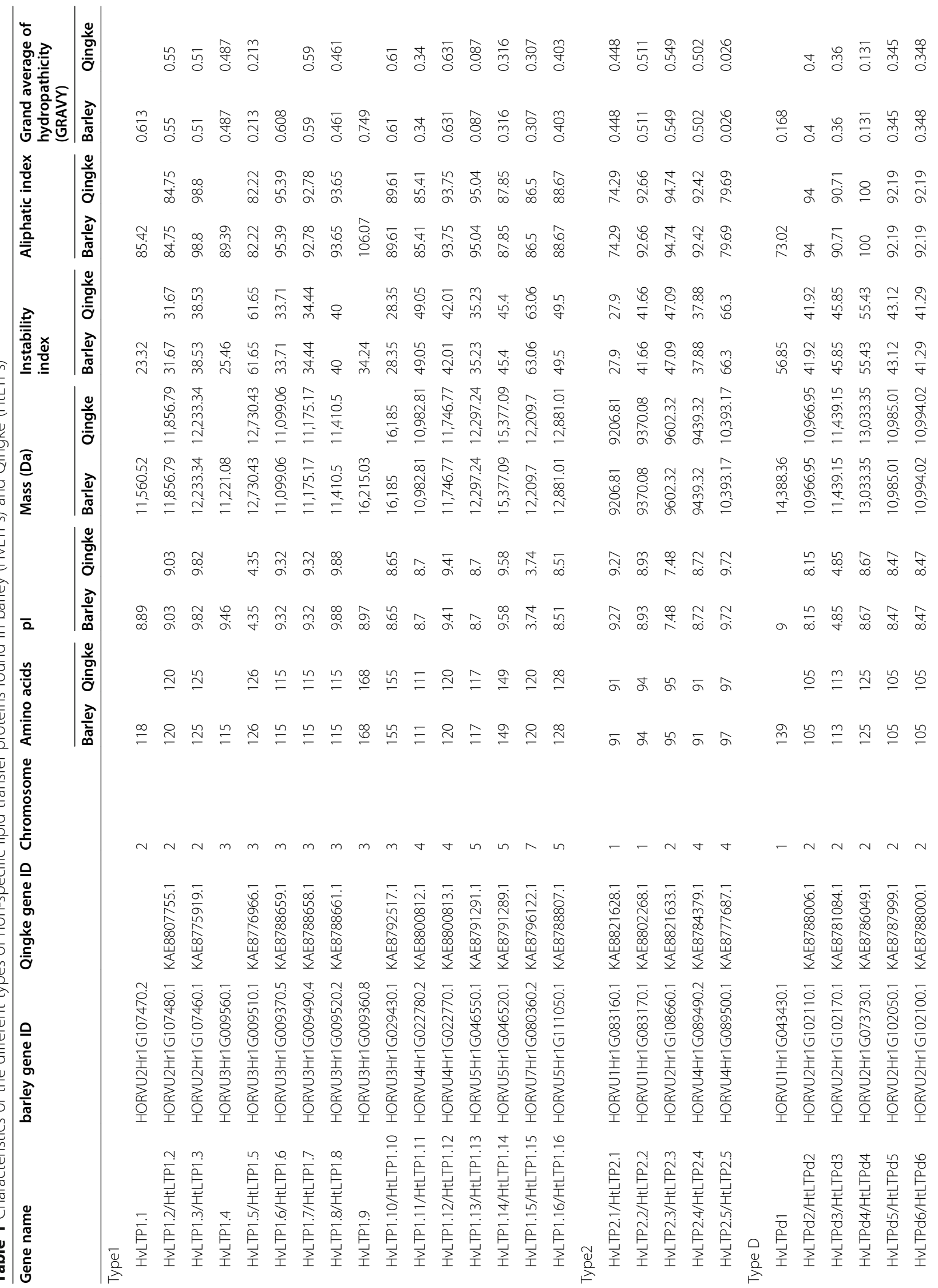




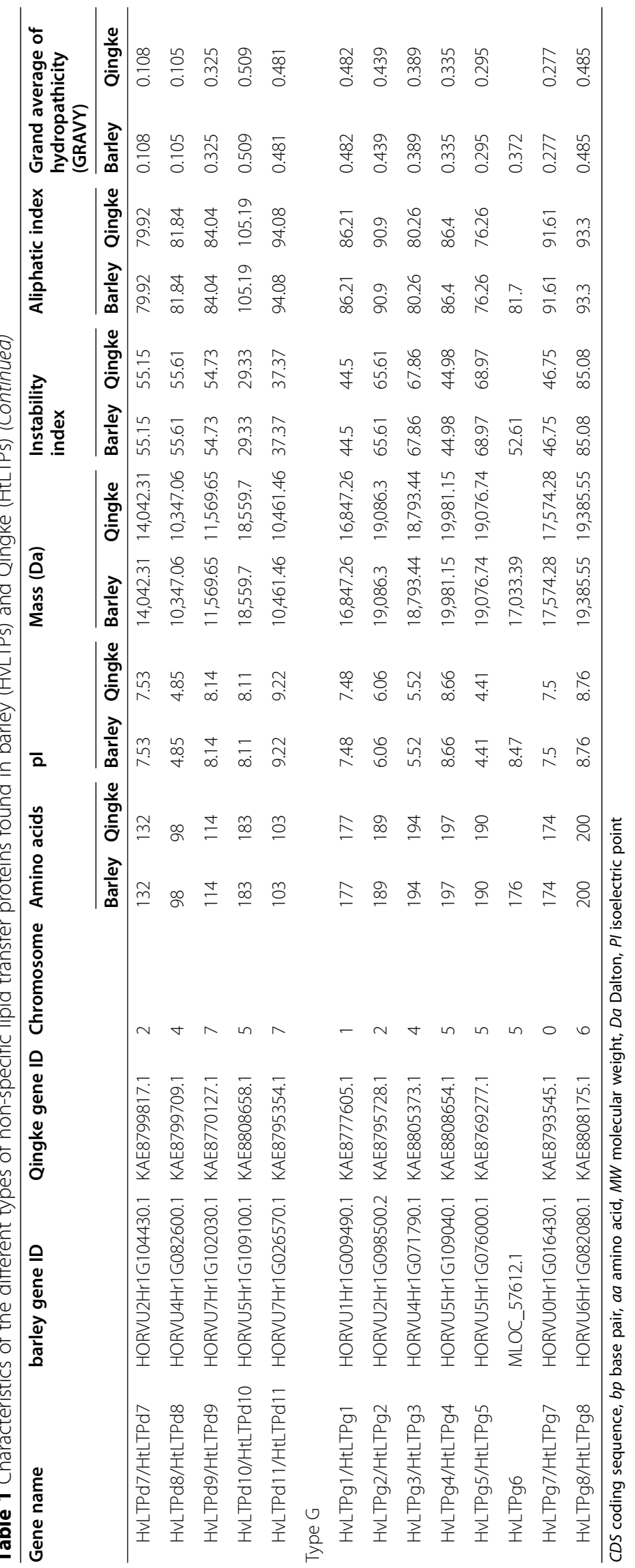


HtLTPs. The results showed that most instability index of HvLTPs and HtLTPs were greater than 40, the most aliphatic indices were greater than 75.5 and the GRAVY value was greater than zero, which indicated that most HvLTPs and HtLTPs were unstable, aliphatic and hydrophobic proteins.

Based on the $\mathrm{Mw}$ of the mature proteins, plant nsLTPs can be classified into two main types: nsLTP1 $(9 \mathrm{kDa})$ and nsLTP2 $(7 \mathrm{kDa})$. Then, according to sequence similarity, Boutrot divided the nsLTPs into nine types (I, II, III, IV, V, VI, VII, VIII and IX) [4]. Recently, plant nsLTPs have been categorized into four major and several minor types $(1,2, C, D, E, F$, $\mathrm{G}, \mathrm{H}, \mathrm{J}, \mathrm{K}, \mathrm{X})$ by intron position, sequence identity and spacing between the cysteine residues in the $8 \mathrm{CM}$, as well as post-translational modifications [41, 42]. Compared with the classification proposed by Edstam, 40 HvLTPs and 35 HtLTPs could be divided into four types, including 16/13(type 1), 5/5(type 2), 11/10(type D) and 8/7(type G) nsLTP genes (Table 1 ). As shown in Table 1, the molecular weights of HvLTPs and HtLTPs are usually between 9206.81 Da and $19,981.15 \mathrm{Da}$, and the molecular weight of Type 2 is smaller than that of the other types, with an average of $9 \mathrm{kDa}$. The molecular weight of the type $\mathrm{G}$ nsLTPs is relatively large and mostly between 16 and $19 \mathrm{kDa}$, with an average of $18 \mathrm{kDa}$. In the type G nsLTPs, the transcripts encode not only a C-terminal signal sequence, but also an $\mathrm{N}$-terminal sequence, thus leading to a post-translational modification which a glycosylphosphatidylinositol (GPI)-anchor is added to the protein to attach the protein to the exterior side of the plasma membrane [43], leading to a much higher molecular weight of the type G nsLTPs than other types.

The main characteristic of plant nsLTPs is the presence of $8 \mathrm{CM}$ motifs. To establish a specific $8 \mathrm{CM}$ consensus for each nsLTP type obtained, we conducted a multiple sequence alignment using the $8 \mathrm{CMs}$ from 40 HvLTPs and 35 HtLTPs. The amino acid sequence alignment of the 8CMs of HvLTPs and HtLTPs revealed a variable number of inter-cysteine amino acid residues. We also found that the alignment results are consistent with the classification results. For the CXC motif, most of the residues at the $\mathrm{X}$ position in type $1 \mathrm{nsLTP}$ are only hydrophilic, while in type 2, D and G nsLTPs, the $\mathrm{X}$ position is usually occupied by hydrophobic residues (Fig. 2). These conserved hydrophobic or hydrophilic

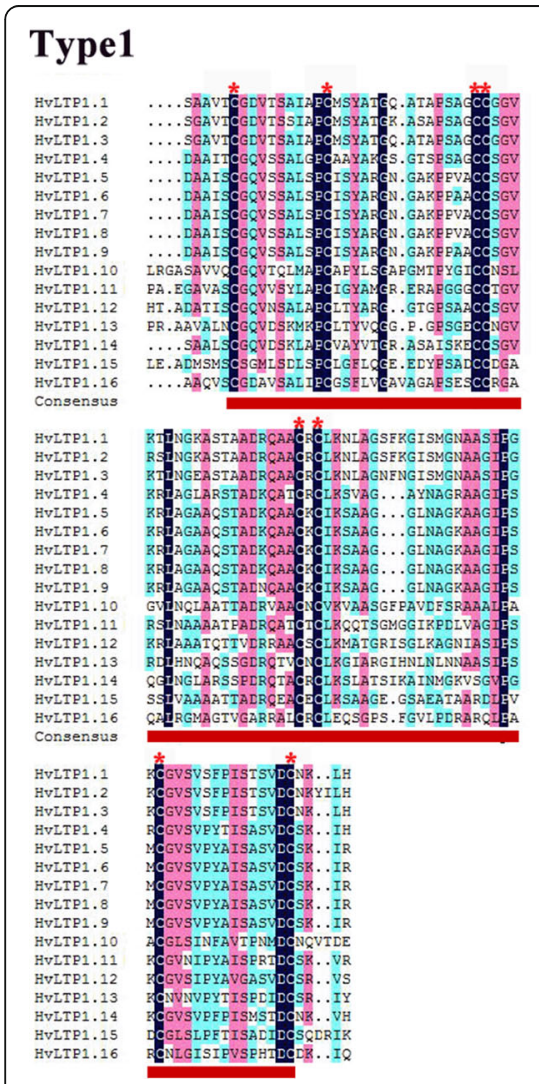

\section{Type2}

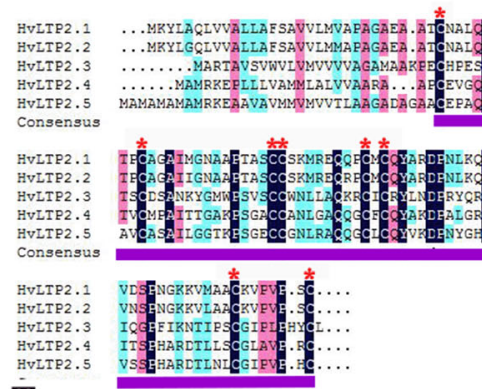

Type g

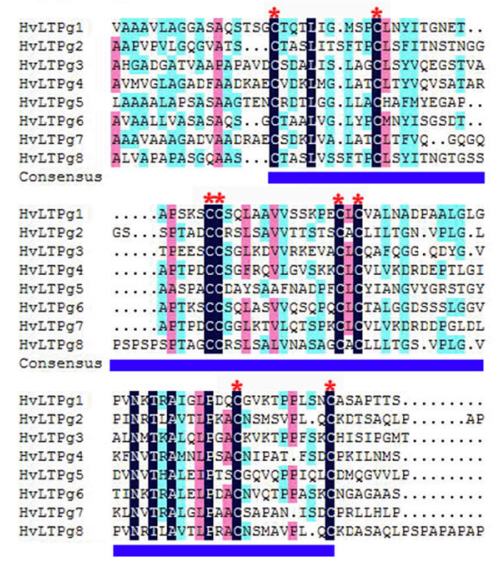

Type d

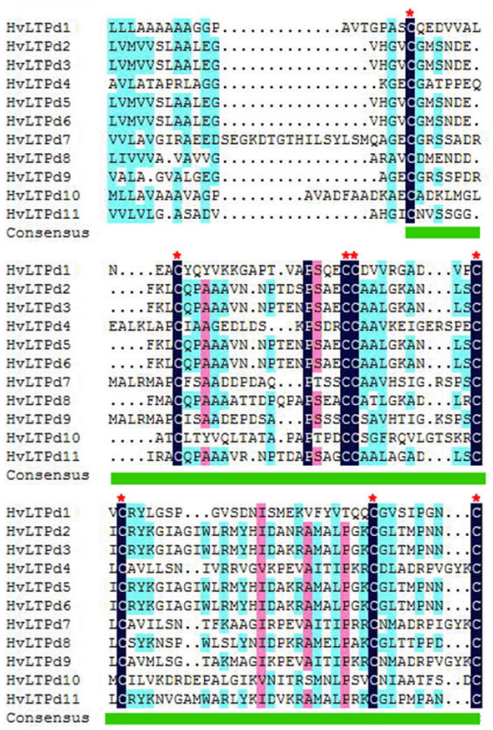

Fig. 2 Multiple sequence alignment of the HvLTP and HtLTP 8CM domain sequences. The horizontal line represents the $8 C M$ domain. * is Eight cyc in $8 \mathrm{CM}$ 
residues may play an important role in the biological function of HvLTPs and HtLTPs and are also consistent with their classification.

\section{Phylogenetic analysis, conserved motifs, and gene structure of the HvLTP and HtLTP families}

To analyse the evolutionary relationship, a phylogenetic tree of 293 nsLTPs from four species, including the HvLTPs and HtLTPs with maize, rice and Arabidopsis, was constructed. Comparing the previous classification data with phylogenetic analysis, it was found that the previous classification and phylogenetic analysis have the same type of nsLTPs; that is, the four groups in the HvLTP and HtLTP classification were consistent with the 1,2, D and G types of the nsLTPs of the other three species, except for sporadic interlaces in types D and G (Fig. 3). The members of types 1 and 2 formed specific clades, indicating that these genes share a common ancestor in major nsLTP types.

Based on the distribution of predicted motifs, 40 HvLTPs and 35 HtLTPs were categorized into four distinct subfamilies, which was consistent with the classification from the phylogenetic analysis (Fig. 4). Both the HvLTPs and HtLTPs have four different subfamilies. Type 1 has a similar motif, and the other types of motif structures are completely different. Common motif 2 was present in all HvLTPs and HtLTPs, except for six genes in type 2 (HvLTP2.1/HtLTP2.1, HvLTP2.2/
HtLTP2.2, HvLTP2.4/HtLTP2.4 and HvLTP2.5/ HtLTP2.5) and type D (HvLTPd8/HtLTPd8 and HvLTPd11/HtLTPd11). Special motifs appear in special types; for instance, motifs 1 and 15 are only present in Type 1, motifs 14 and 16 are only present in Type D, and motif 13 only exists in Type G HvLTPs and HtLTPs.

As a type of evolutionary relic, the intron-exon arrangement carries the imprint of gene family evolution. The gene structures of the HvLTPs and HtLTPs were also investigated (Fig. 5). Investigation of the HvLTP and HtLTP gene structures revealed a low diversity distribution of intronic regions amid the exonic sequences. 40 HvLTP and 35 HtLTP genes were predicted to be interrupted by $0-2$ introns positioned -9 to $104 \mathrm{bp}$ downstream of the codon encoding the eighth cysteine in 8CM (Table 1). Additionally, it was interesting to find a similar exon/intron pattern in each group. For instance, the HvLTP and HtLTP genes in type 2 lack introns while the type $G$ contains 2 introns. Except for HvLTPd1, HvLTPd7/HtLTPd7, and HvLTPd9/HtLTPd9, no introns were detected in the coding regions of type $\mathrm{D}$ genes.

\section{Chromosomal localization and gene duplication of HvLTPs and HtLTPs}

After determining the genomic location information of 40 HvLTP and 35 HtLTP genes, the genomic location

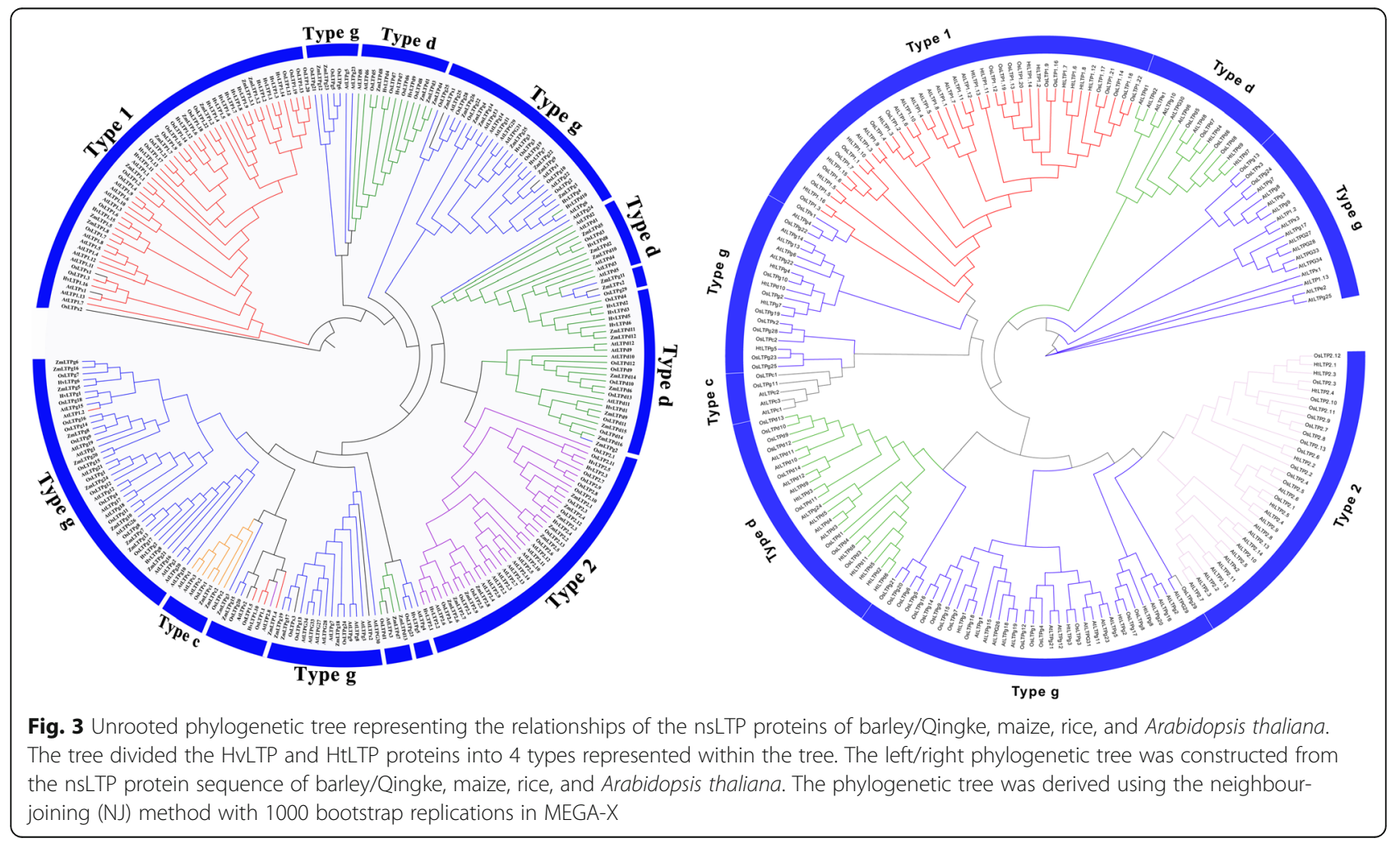




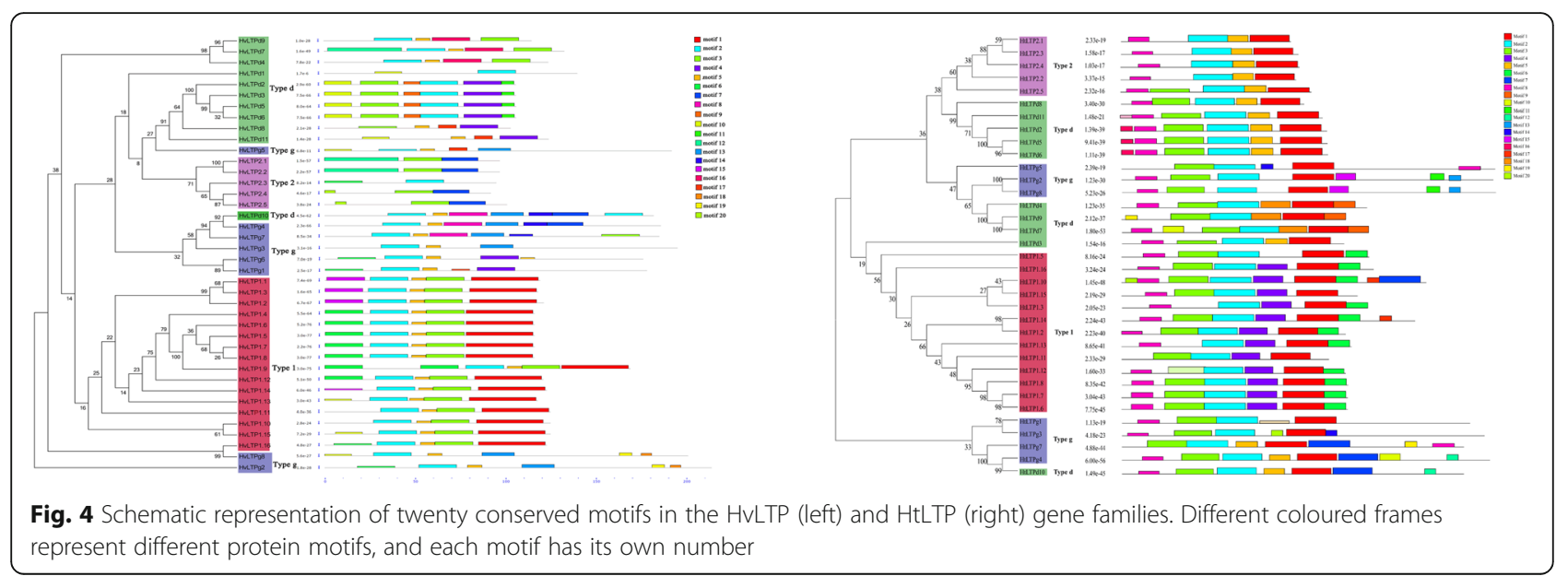

information belonging to the 2012 IPK genes was not found for one of them (MLOC_57612.1). The Chromosomal localization results showed that 39 HvLTP and 35 HtLTP genes were unevenly distributed on 7 chromosomes of barley and Qingke, and there were obvious HvLTP and HtLTP gene clusters. The maximum number of HvLTP and HtLTP genes was contained on chromosome 2 (11), and the minimum number (1) was shown on chromosome 6 (Fig. 6).

Gene duplication is generally considered to be a major driving force in evolutionary innovation and leads to genomic complexity. In this study, six tandem repeats were identified in the barley genome (Table S2), which was consistent with obvious $H v L T P$ gene clusters in the barley chromosome. Two significant clusters were found on chromosomes 2 and 3. In addition, one sister pair appeared to be generated from segmental duplication (Table S2, Figure S1A). Furthermore, we analysed the collinearity of HvLTPs with nsLTP genes in rice and wheat. Eleven out of $39 H v L T P$ genes had collinear genes with rice, while $22 H v L T P$ genes had syntenic members between barley and wheat (Figure S1B).

\section{Promoter and stress expression analysis of HvLTPs and HtLTPs}

Plant nsLTPs display a complex tissue-specific and developmental expression patternand are mainly expressed in the tapetum, pericarp and epidermal cells of embryos, stems, leaves and roots [42-44]. According to on the RPKM values of each $H v L T P$ gene published on the IPK website, an expression heatmap of $40 H v L T P$ genes in the 16 different tissues were mapped (Fig. 7A). The results showed that the HvLTPs had distinctly different expression patterns during different developmental stages as well as in different plant tissues. All HvLTPs were not expressed in INF1, and the expression level of HvLTPs in INF2 was also extremely low. Among the different $H v L T P$ types, the relative expression levels of type 1 and type 2 genes were higher and more specific than those of type D and type G genes, except that the expression

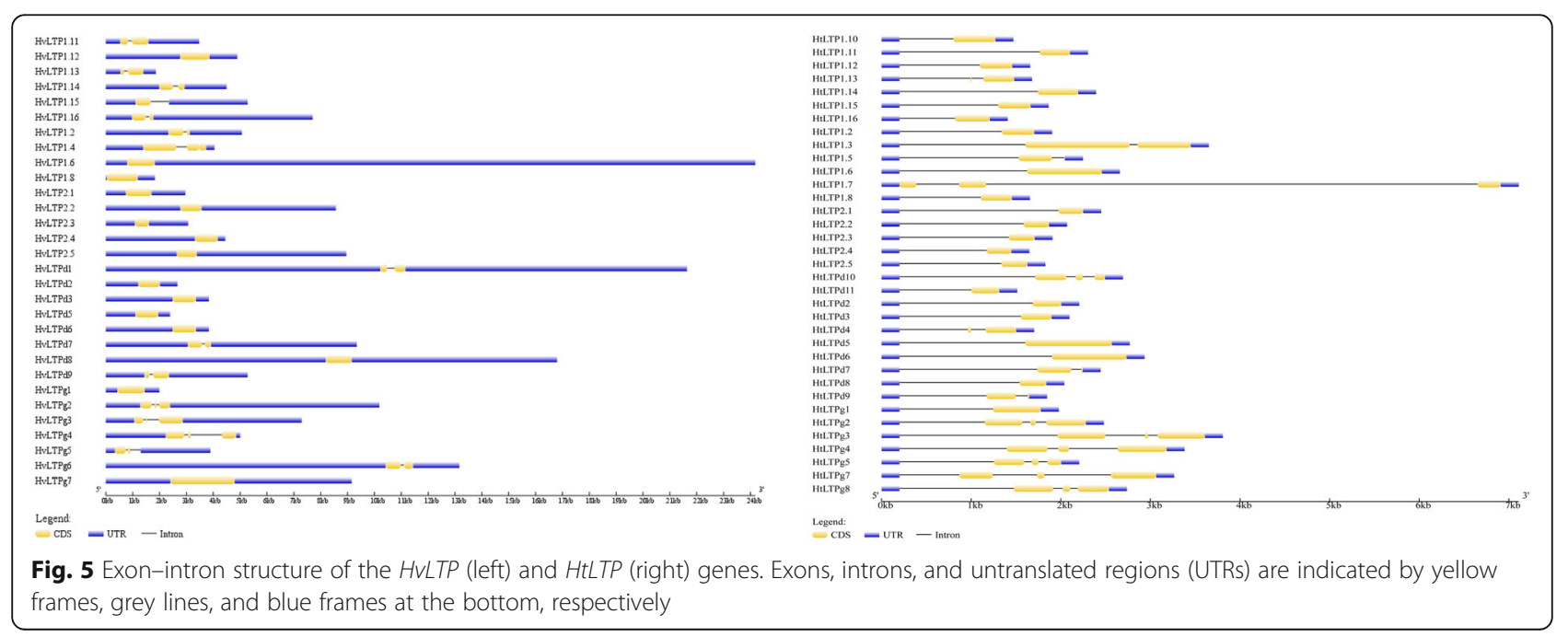




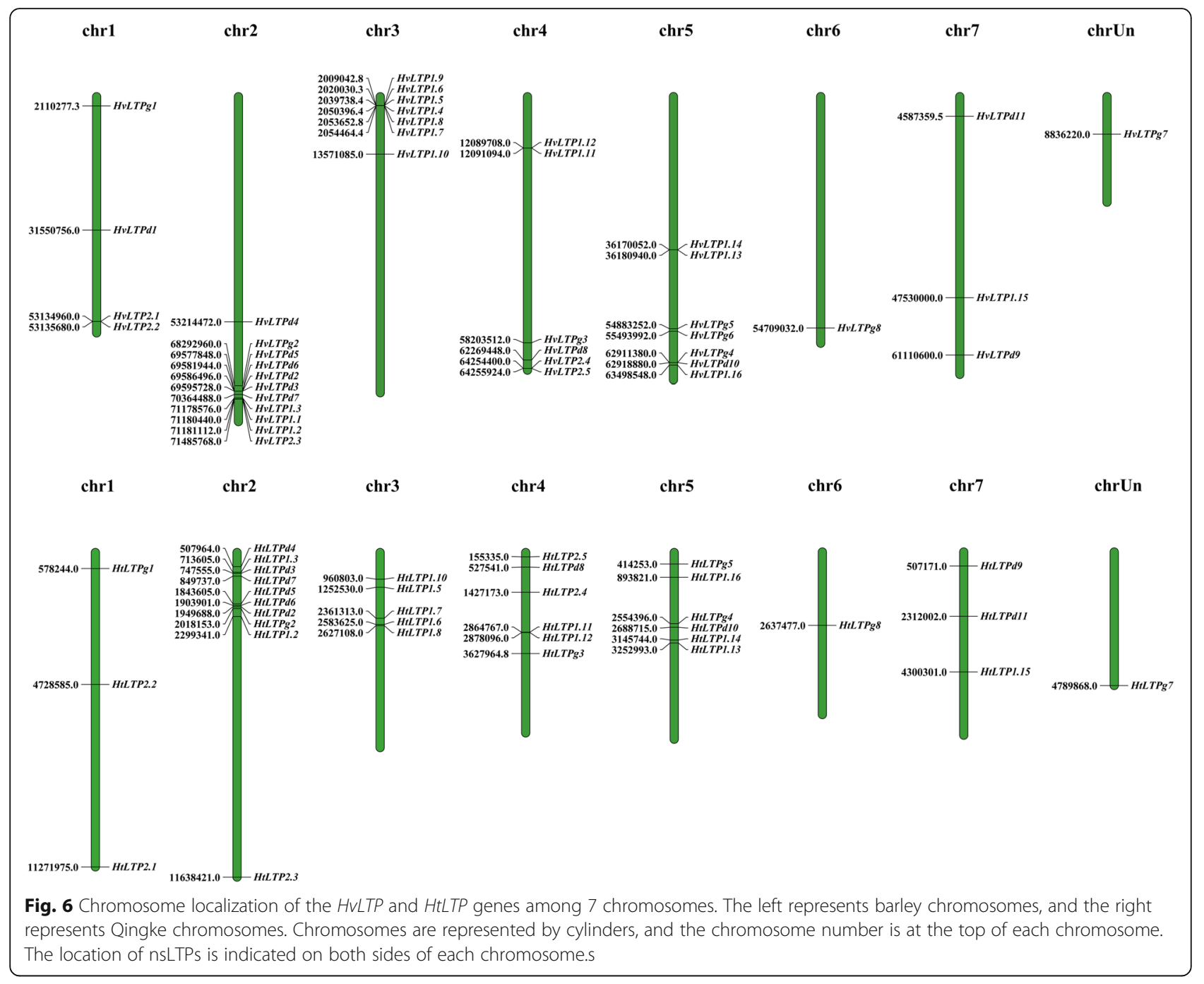

of HvLTP1.10 was lower in all tissues. Type 1 and type 2 HvLTP genes showed obvious tissue-specific expression patterns. For example, $H v L T P 1.2$ is mainly expressed in LEA. HvLTP1.13 is specifically expressed in CAR15, and $H \nu L T P 2.4$ and $H v L T P 2.5$ are specifically highly expressed in CAR5 and CAR15, which indicates that these three genes might be involved in grain development. Given the high expression level of barley type 1 HvLTPS in the above transcriptome data, we simultaneously detected the tissue expression patterns of 10 type 1 barley and Qingke nsLTP genes in five different tissues by qRT-PCR (Fig. 7B). The results showed that the expression levels of type 1 LTP genes in barley and Qingke were very low in the roots, and the expression pattern of barley type $1 H \nu L T P s$ was basically consistent with that of transcriptome data. However, the expression patterns of 10 nsLTP genes were different in the barley and Qingke tissues. For example, the expression level of barley LTP1.4 was higher in the leaves, while that of Qingke LTP1.4 was lower in the leaves. The expression level of barley LTP1.11 was higher only in the seeds, and that of Qingke LTP1.11 was higher in the stems and flowers.

An in silico analysis of the $1.5 \mathrm{~kb}$ upstream region (starting from the translation initiation site) of $35 \mathrm{HvLTP}$ and HtLTP genes revealed various regulatory elements associated with development and abiotic or biotic stress signalling (Fig. 8.). In addition to the TATA-box and CAAT-box, the A-box is the most common type of ciselements in the HVLTP and HtLTP genes. In this study, we found that the cis-elements of the HvLTP and HtLTP genes included stress response elements (ARE, MBS, MYB, LTR, TC-rich motif, and DRE), hormone-related elements (ABRE, TGACG-element, CGTCA-motif, TGA-element, TCA-element, GARE-motif, P-box, TATC-box, AuxRR-core, ERE, and Wbox), indicating that HVLTP and HtLTP are involved in stress response and hormone signalling. Additionally, the regulatory element involved in light responsiveness also appears to be enriched in the HVLTP and HtLTP genes, including G-Box, ACE, Box 4, GT1-motif, Sp1, TCT-motif, 

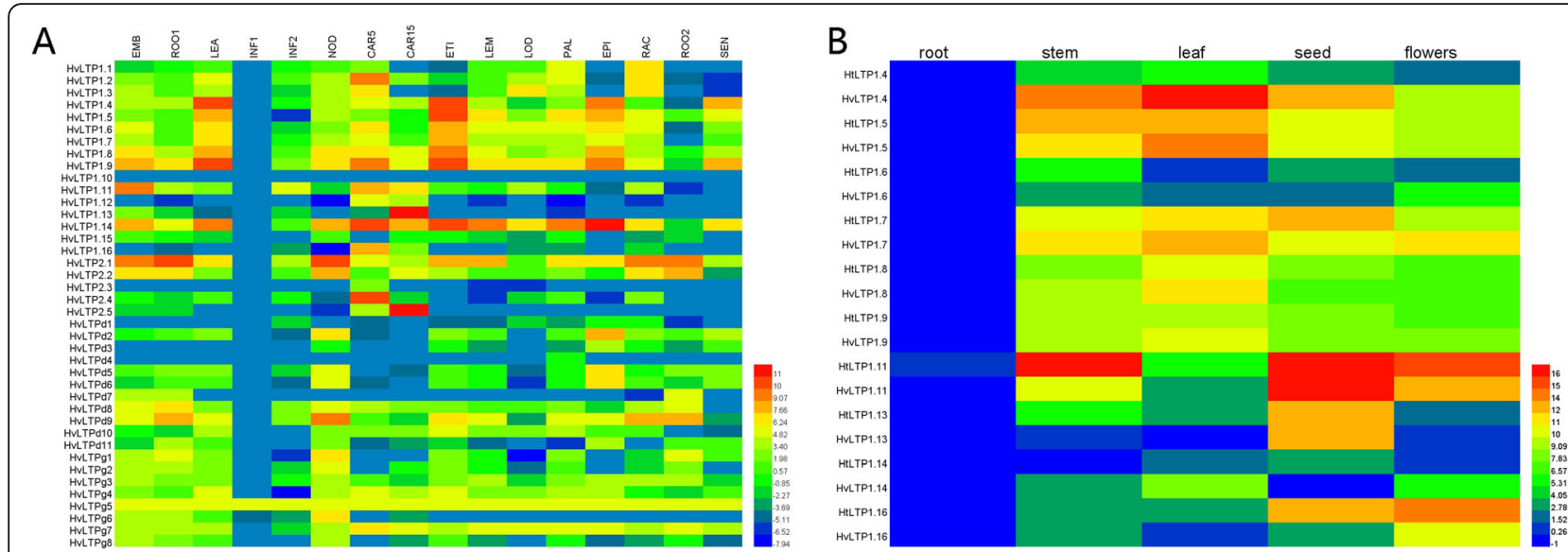

Fig. 7 Tissue expression pattern in the HvLTP (left) and HtLTP (right) genes. On the left, the data were obtained from the BARLEX database at the Leibniz Institute of Plant Genetics and Crop Plant Research (IPK). X-axis: mRNA levels in 16 different tissues and life stages of barley. EMB: 4-day embryos; ROO1: roots from seedlings (10 cm shoot stage); LEA: shoots from seedlings (10 cm shoot stage); INF1: young developing inflorescences (5 mm); INF2: developing inflorescences (1-1.5 cm); NOD: developing tillers, 3rd internode (42 DAP); CAR5: developing grain (5 DAP); CAR15: developing grain (15 DAP); ETI: etiolated seedling, dark cond; LEM: Inflorescences, lemma (42 DAP); LOD: Inflorescences, lodicule (42 DAP); PAL: Dissected inflorescences, palea (42 DAP); EPI: Epidermal strips (28 DAP); RAC: Inflorescences, rachis (35 DAP); ROO2: Roots (28 DAP); and SEN: Senescing leaves (56 DAP). On the right, the data were obtained from the qRT-PCR results. X-axis: mRNA levels in 5 different tissues of Qingke. Different mRNA levels of each putative HvLTP and HtLTP are given as colour codes. Blue indicates a low expression level, and red indicates a high expression level

GATA-motif, I-box, AE-box, Box II, and TCCC-motif. Some HvLTP and HtLTP genes have specific developmental response elements such as zein metabolism regulation $\left(\mathrm{O}_{2}\right.$-site), meristem expression (CAT-box), and seed-specific regulatory elements (RY-elements). A comparison of the 35 gene promoters of HvLTPS and HtLTPs showed that only 6 genes had the same ciselement organization, indicating that there were more stress response- and hormone-related elements in Qingke. There are both similarities and differences between HvLTPs and HtLTPs. Similarly, all have a similar number of stress response elements, most of which have AREs (essential for the anaerobic induction), LTRs (involved in low-temperature responsiveness) and MBSs (MYB binding sites involved in drought-inducibility). For nsLTP, MBS has been previously reported as a target

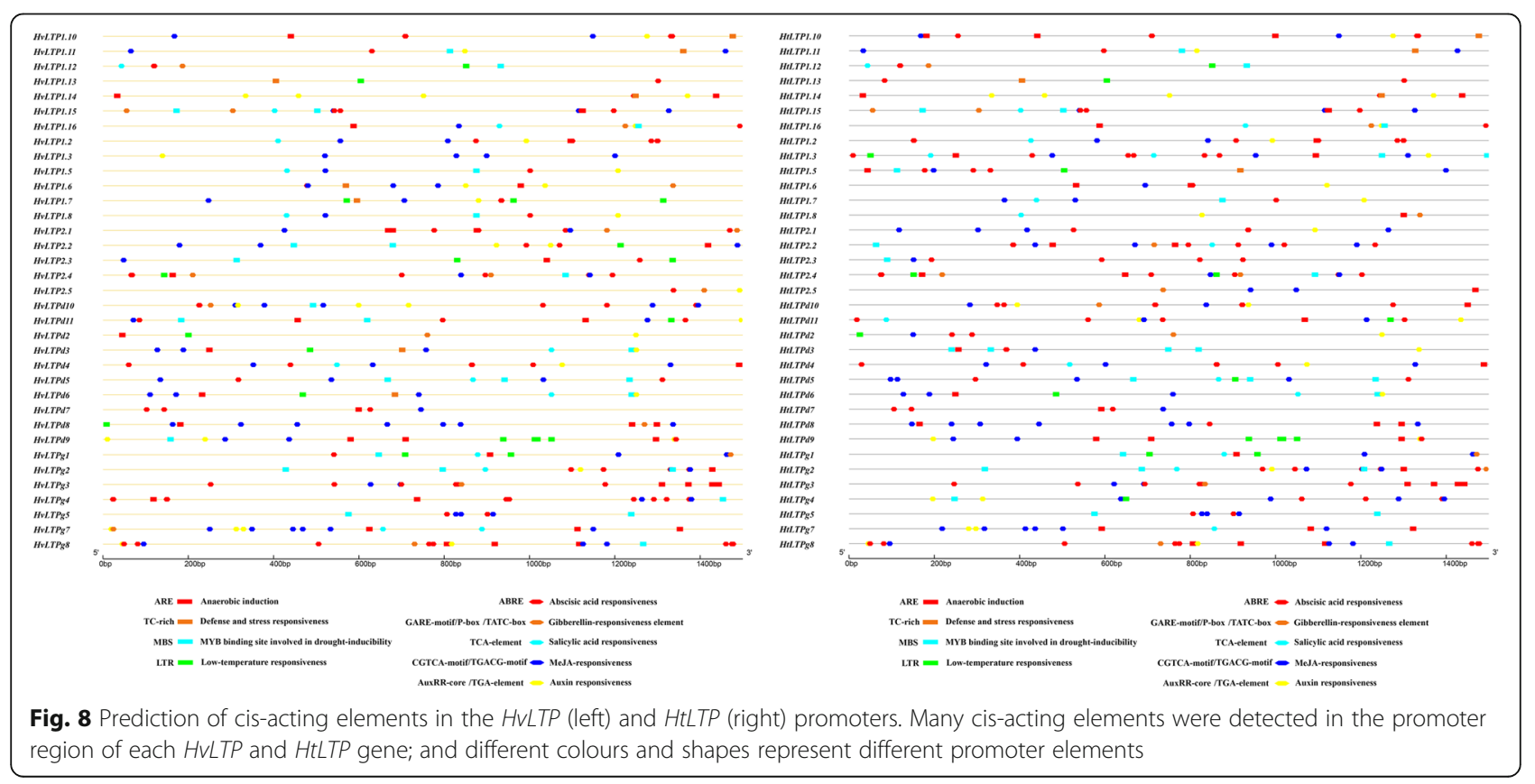


for MYB transcription factor to modulate plant tolerance to freezing and drought stress [45], suggesting that these HvLTPs and HtLTPs containing MBS or MYB elements may participate in abiotic stress signalling of MYB. The difference is that the number of hormone response elements and light responsive elements in Qingke was higher than in barley, and ABRE and G-box are the most important and different hormone and light-responsive regulatory elements in HvLTP and HtLTP genes, which may mean that Qingke may rely on the abscisic acid hormone pathway and G-box related light-response pathway to adapt to more severe plateau environment [39].

qRT-PCR was performed further to explore the expression patterns of $16 H v L T P$ and 7 HtLTP genes in the root and leaf tissue under abiotic stress, which means that barley seedlings were treated for 2 days with drought, cold and salt stress, and then recovered for 2 days (Fig. 9, Figure S2). Similar to spatiotemporal tissue expression of $H v L T P S$, the expression level of Type 1 genes is higher than that of other types in HvLTPs under abiotic stress; therefore, the expression of type 1 genes of HtLTP genes were also assessed by qPCR assay. The seven Type 1 HvLTP and HtLTP genes had significant responses in the leaves under cold, drought and salt stress, but no or low responses in the roots, which was consistent with the low expression in the root in the IPK database (Fig. 9). The expression level of all barley Type 1 HvLTP genes was decreased under cold stress, while four HtLTP genes were upregulated in Qingke. The 4 Type $1 H v L T P$ and HtLTP genes were upregulated in barley and Qingke under drought stress, but two of them were different. For example, the expression of HvLTP 1.7 was downregulated in barley, but HtLTP 1.7 was upregulated in Qingke; and the expression of $H v L T P$ 1.3 was upregulated in barley, but HtLTP 1.3 was downregulated in Qingke. Only one Type 1 HvLTP gene was upregulated in barley under salt stress, while six Type 1 HtLTP genes were upregulated in Qingke. In general, the expression patterns of seven Type 1 HvLTP and HtLTP genes in barley and Qingke under abiotic stress were different. After abiotic stress was removed, the change trend of the type 1 gene was also different

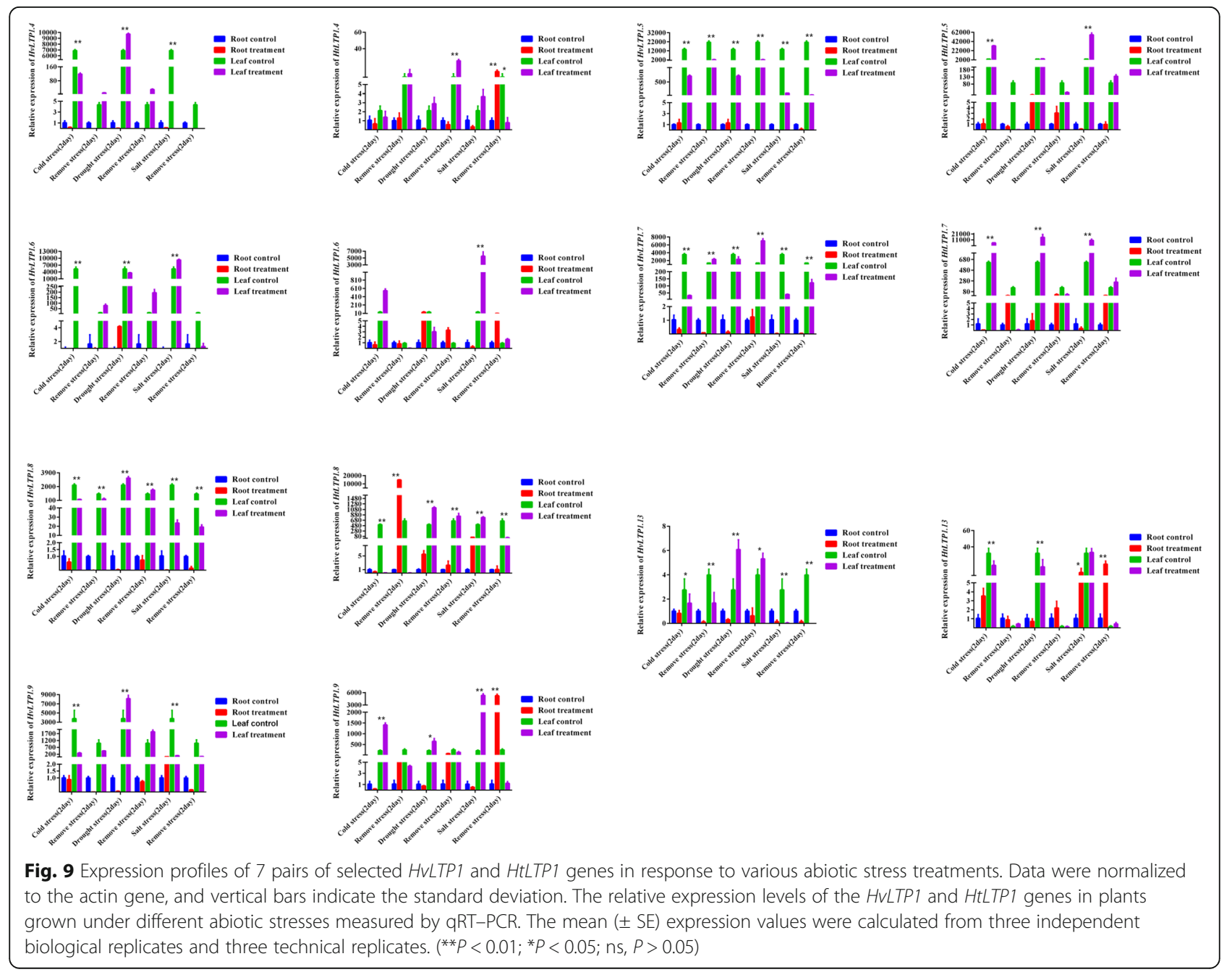


between barley and Qingke. For example, there were 3 genes in barley while there were 6 genes with opposite change trend in Qingke under cold stress; there were 5 genes in barley but 4 genes with the same change trend in Qingke under drought stress; and there were 6 genes in barley but only 3 genes with the same change trend in Qingke under salt stress. At the same time, there were also differences in genes with opposite trends; for example, the HvLTP 1.4 and $H v L T P 1.6$ genes were downregulated first and then upregulated under cold stress in barley, while HtLTP 1.4 and HtLTP 1.6 were upregulated first and then downregulated in Qingke.

Unlike type 1, two genes (HvLTP2.1/HvLTP2.2) of type 2 responded significantly to drought, cold and salt stresses in the roots and HvLTP2.1 responded significantly to salt stress in the leaves. The two genes showed the same change trends in the roots under the removal of abiotic stresses basically. For example, the two genes were upregulated continuously under cold stress and after the removal of cold stress, but they were downregulated under drought stress and upregulated after the removal of drought stress; moreover, slightly different were observed under salt stress. HvLTP2.1 was upregulated continuously with and without salt stress, while the HvLTP2.2 gene changed in opposite directions, i.e., it was downregulated first and then upregulated. Type D genes were downregulated under cold and drought stress, while most type D genes were upregulated under salt stress in the roots. The HvLTPd2 and HvLTPd5 genes also showed significant effects and were upregulated in the leaves under drought and salt stress. After the removal of abiotic stress, the overall fluctuation of type D genes was the opposite, but the HvLTPd7 gene showed the same trend. In type G, the response of HvLTPg1 in the roots was upregulated significantly under cold stress, while the response in the leaves was upregulated significantly under drought and salt stress. $H v L T P g 2$ was downregulated significantly in roots and leaves under the three abiotic stresses. Only $H v L T P g 5$ was upregulated significantly in leaves under cold stress. In general, the type $G$ genes were not affected due to low expression after removal of abiotic stress, and only some genes had the opposite changing trends, including the HvLTPg1 genes in roots and HvLTPg2 genes in roots and leaves under drought stress; and the HvLTPg5 gene in leaves under the three abiotic stresses (Figure S2).

\section{Discussion}

Plant nsLTPs are a large transporter family composed of 79 members in Arabidopsis, 77 in rice, 63 in maize, 58 in sorghum, 63 in cabbage and 156 in wheat, all of which are classified as different types [4, 17, 38, 39, 46]. In 2018 , the wheat nsLTP family was comprehensively analysed again, and 461 putative TaLTPs were identified from the whole wheat genome [47]. Edstam provided comprehensive information about the categorization of the nsLTP gene family based on phylogenetic clustering and facilitated further functional analysis [41]. In this study, 40 and 35 nsLTPs were identified in the barley and Qingke genomes and classified into 4 subfamilies (Type 1, 2, D and G) following Edstam's classification (Table 1, Fig. 2). In addition, the number and classification of HvLTPs were also compared with those in Arabidopsis, rice, maize and cabbage (Table 2). The results showed that the total number of nsLTP gene families in barley and Qingke was less than that in other species. Moreover, type $\mathrm{E}$ is unique to dicotyledons, while HvLTPs and HtLTPs are also deficient in type $\mathrm{C}$ and type X (Table 2). Meanwhile, the proportion of nsLTPs in each subfamily indicated that type $G$ seemed to have contracted in barley, but no expansion was observed. Therefore, the decrease in nsLTP gene number in barley may be due to the loss of individual types and the reduction of individual populations. In addition, this number may be due to the inhibition of gene expansion. Zhang (2019) showed that 70 barley nsLTP genes were identified by keyword searching in Phytozome v12.1.6 and divided into five types $(1,2, C, D$, and G) [40], which indicates that previous research results of this barley nsLTP gene family expansion are different from ours. Compared with Zhang's research, our research collected more comprehensive data through a BLASTP search, HMM search and keyword search of the barley genomes, including in the IPK, Ensembl, NCBI and Phytozome databases, and obtained 160 potential HvLTP genes. Referring to previous screening methods [17], the screening method was also more stringent and finally identified 40 barley HvLTP genes; therefore, our results were more accurate, comprehensive and reliable.

The role of gene duplication in the origin of evolutionary novelty and complexity has long been recognized [48]. In our study, we found one segmental duplication and six tandem duplication events in the barley nsLTP gene family (Table S2; Figure S1). These results suggest that gene expansion is inhibited overall and the evolution of the barley nsLTP gene family not only involves gene retention but also gene loss and mutation. The retention and loss of genes may be associated with related functions during plant evolution [49]. In addition, these paralogous duplicated genes may retain some essential functions in subsequent evolution. For example, five pairs of tandem duplication genes (HvLTP1.1/ HvLTP1.2/HvLTP1.3, HvLTP1.5/HvLTP1.7/HvLTP1.8, HvLTP1.6/HvLTP1.9, HvLTP2.1/HvLTP2.2, HvLTPd2/ HvLTPd5/HvLTPd6) shared similar expression profiles. However, one pair of duplicated genes (HvLTP2.4/ HvLTP2.5) showed significant divergence in expression. At the same time, another pair of segmental duplication 
Table 2 Numbers of nsLTP genes in different species

\begin{tabular}{lllllllll}
\hline species & Total number of members & Type 1 & Type 2 & Type C & Type D & Type E & Type G & Type X \\
\hline Hordeum vulgare & 40 & 16 & 5 & 0 & 11 & 0 & 8 & 0 \\
Qingke & 35 & 13 & 5 & 0 & 10 & 0 & 7 & 0 \\
Arabidopsis thaliana & 79 & 13 & 13 & 3 & 12 & 2 & 33 & 3 \\
Oryza sativa & 77 & 18 & 13 & 2 & 14 & 0 & 27 & 3 \\
Zea mays & 63 & 8 & 9 & 2 & 15 & 0 & 26 & 3 \\
Brassica oleracea var. capitata & 89 & 19 & 12 & 1 & 18 & 2 & 28 & 9 \\
\hline
\end{tabular}

genes (HvLTP1.12/HvLTP1.13) also showed different expression patterns. The differential expression patterns of these duplicated genes in barley indicated that these genes may be functionalized after duplication events during the evolutionary process, leading to significant variation in gene regulation [50,51].

Sufficient evidences has been obtained to show that nsLTPs are involved in various types of stress resistance, including resistance to phytopathogens, freezing, drought and salt [42, 52]. In our study, the stressdependent cis-elements in the promoter regions of the HVLTP and HtLTP genes were analysed. The results showed that the promoters of the HvLTP and HtLTP genes contained stress response elements (STRE, DRE, MBS, MYB, TC-rich repeats) and hormone-related elements (ARE, LTR, ABRE, ERE, TCA-element, TGAelement, TGACG-element, CGTCA-motif, and W box), indicating that the HvLTPS and HtLTPs are involved in the stress response. Meanwhile, the expression patterns of 17 HvLTP genes in response to abiotic stresses also demonstrated their correlation with abiotic stresses. In general, the response of these genes in the root was weaker than that in the leaf. Under different abiotic stresses, the response levels of different type members were inconsistent. For example, the response of $H v L T P$ type 1 was significantly higher than that of the other subfamilies. The blt4 gene of barley is a low-temperature response gene with different responses to drought, pathogen attack, and abscisic acid (ABA) [28]. Previous studies have shown that blt4 belongs to the barley nsLTPs located on chromosome 3 and can act as a regulatory protein to stabilize plasma membrane activity and resist low-temperature injury [12]. Interestingly, blt4 genes belong to the barley nsLTP type 1 , and some of them appear in tandem duplication events, such as blt4.3 (HvLTP1.8)/blt4.9 (HvLTP1.7) and blt4.1 (HvLTP1.6) (Table S2, Fig. 6). The expression patterns of these tandem duplication genes were similar, indicating that these genes may retain the same function and coordinate the regulation of the stress response through the tandem repeat of gene clusters.

Different promoter cis-elements and their epigenetic changes have been reported to affect gene regulation, thereby resulting in different gene expression levels and further affecting adaptation to the environment, including altitude changes [53-55]. We also compared the nsLTP gene promoter and stress expression pattern between barley and Qingke and found that the nsLTP gene sequences between barley and Qingke were basically the same, but the promoter and stress expression pattern were different. Moreover, the expression of nsLTP gene was directly related to its promoter, which indicated that the stress expression pattern of nsLTP gene was changed by its promoter. Combined with the analysis of upstream cis-elements of HvLTPs and HtLTPs and their expression patterns under abiotic stress, it was found that a large number of HvLTP and HtLTP genes may change their regulatory modes due to different upstream ciselements and cause different abiotic stress responses. Generally, there is little difference between them in terms of the HVLTP and HtLTP genes themselves, including the gene structure, conserved motifs, phylogenetic analysis and classification. The main difference between them lies in its promoter regulatory elements, which may lead to the difference of expression patterns between HvLTP and HtLTP genes, thus leading to the adaptability of Qingke to extreme plateau climate.

\section{Conclusions}

In summary, 40 HvLTPs and 35 Qingke nsLTPs were identified in barley and Qingke in this study. A comprehensive study of HvLTPs and HtLTPs will reveal important features of the nsLTP gene family, such as gene structure, evolution, chromosome distribution, conserved motifs, segmental and tandem duplication, upstream cis-elements, and stress expression patterns. The study results could be considered a useful source for future nsLTP gene research in either barley or Qingke or for comparisons of different plant species. This study will help to provide a foundation for future research on the molecular mechanisms of barley and Qingke stress adaption.

\section{Materials and methods}

Plant materials, growth conditions and abiotic stress treatment

The barley variety 'Morex' and Qingke variety 'Dulihuang' were used as the plant material in this study. The 
seeds were surface sterilized with $10 \% \mathrm{H}_{2} \mathrm{O}_{2}$ (v/v) for 10 min and rinsed with deionized water for several times. Then, the soaked seeds were kept at $25^{\circ} \mathrm{C}$ for $48 \mathrm{~h}$ in darkness to germinate in a light growth chamber. The germinating seeds were planted in $1.5 \mathrm{~L}$ pots, filled with Hoagland's nutrient solution and were grown in a greenhouse at $22^{\circ} \mathrm{C}$ with a photoperiod of $16 \mathrm{~h}(12,000 \mathrm{~lx})$ and a dark period of $8 \mathrm{~h}$. Seedlings were grown to maturity (14 days of germination) under normal conditions and then treated under different abiotic stress conditions including drought, cold and salt. For salt stress, $200 \mathrm{mM}$ $\mathrm{NaCl}$ was added to the nutrient solution for $48 \mathrm{~h}$; for drought stress, $18 \%$ polyethylene glycol was added to the nutrient solution for $48 \mathrm{~h}$; and for cold stress, the seedlings were kept at $4^{\circ} \mathrm{Cfor} 48 \mathrm{~h}$. Then, tissue samples composed of leaves and roots from every stress treatment were collected once. After $48 \mathrm{~h}$, the seedlings were transferred to nutrient solution without stress and cultured for $48 \mathrm{~h}$ in the growth chamber. Then, the tissue samples were taken again. Then, the plant materials were collected and immediately frozen in liquid nitrogen for RNA extraction. All samples were replicated three times.

\section{Identification of putative nsLTP genes in the barley and Qingke genomes}

All known nsLTP amino acid sequences from Arabidopsis (A. thaliana), maize (Zea mays L), cabbage (Brassica rapa $\mathrm{L}$ ) and rice (O. sativa) were used as queries (Table S1) by searching against the barley database using the BLASTP (IPK) program with the default parameters (http://webblast.ipk-gatersleben.de/barley_ibsc/). Simultaneously, an HMM search was performed on the barley genome release-41 from Ensembl Genomes (http:// ensemblgenomes.org/), and amino acid sequences containing the domain PF00234 (Tryp alpha amyl domain, plant lipid transfer/seed storage/trypsin-alpha amylase inhibitor) were obtained. In addition, the proteins associated with nsLTP were searched by keywords from the NCBI (https://www.ncbi.nlm.nih.gov/), IPK (http://apex. ipk-gatersleben.de/apex) and Phytozome (https:// phytozome.jgi.doe.gov/pz/portal.html) databases. The results from the BLASTP, HMM and keyword searches were combined to remove redundant sequences. To increase the probability of detecting putative nsLTPs in barley, all barley protein sequences were downloaded from the NCBI and IPK databases and submitted to the BatchWeb CD-Search Tool (http://www.ncbi.nlm.nih. gov/Structure/cdd/wrpsb.cgi) to verify the presence of nsLTP domain cl07890(PF00234 belong to cl07890). Pfam (http://pfam.sanger.uk/) validation was then performed using the domain PF00234. Then, the deduced protein sequences of candidate nsLTPs were manually examined to determine whether they harboured the
8CMs $(\mathrm{C} \ldots \mathrm{C} \ldots \mathrm{CC} \ldots \mathrm{CXC} \ldots \mathrm{C} \ldots \mathrm{C})$, and proteins lacking the essential cysteine residues were removed. Subsequently, the proteins without NSSs (N-terminal signal sequence prediction, http://www.cbs.dtu.dk/ services/SignalP, checked by the PROTTER http://wlab. ethz.ch/protter/start/) were also removed, and the remaining C-terminal glycosylphosphatidylinositol (GPI) anchors (GPI anchor signal prediction, http://mendel. imp.ac.at/gpi/plant_server.html and http://psort.hgc.jp/ form.html) remained. Subsequently, putative prolinerich or hybrid proline-rich proteins, which are characterized by a high proportion of proline, histidine and glycine residues in the sequence located between the NSS and the $8 \mathrm{CM}$, were excluded from further analyses. The protein sequences of At2S1-At2S4 and RAT1 were then BLAST-searched against the rest of the candidate nsLTP proteins to exclude possible inhibitors and cereal storage proteins. Proteins with more than 120 amino acids at maturity were also discarded, and the final remaining amino acid was identified as the target amino acid sequence, named HvLTPs (the nsLTPs in barley). The entire screening process was undertaken strictly according to Fig. 1.

The Qingke (Tibetan hulless barlay) genome was downloaded from the NCBI (https://ftp.ncbi.nlm.nih. gov/genomes/genbank/plant/Hordeum_vulgare/all_ assembly_versions/GCA_004114815.1_Hulless_Barley_ ass.V2/), and putative Qingke nsLTPs Orthologues were identified using HvLTP sequences by local BLAST and named HtLTPs (nsLTPs in Qingke).

\section{Multiple sequence alignment and classification}

The amino acid sequences of the putative 40 HvLTPs and 35 HtLTPs were downloaded and multiple alignment of the $8 \mathrm{CM}$ part of these sequences was then conducted and manually edited using the DNAMAN program. Additionally, the amino acid sequences were submited to the online site Compute $\mathrm{pI} / \mathrm{Mw}$ tool (http://web.expasy.org/compute_pi/) to calculate the isoelectric point and molecular weight. Sub-cellular localization of these genes was predicted by the Plant-mPLoc online service (http://www.csbio.sjtu.edu. cn/bioinf/plant-multi/). The three-dimensional structures of all putative HvLTPs and HtLTPs were also predicted by SWISS-MODEL (https://swissmodel. expasy.org).

The HvLTPs and HtLTPs can be divided into four major and several minor types according to sequence identity, spacing between the Cys residues and intron position in the $8 \mathrm{CM}$, and the post-translational modifications based on the presence of a GPI modification site. In the second round of classification, the HvLTPs and HtLTPs were sorted based on the 
identity matrix calculated from the multiple sequence alignments [41].

\section{Phylogenetic construction}

The nsLTP amino acid sequences of Arabidopsis, maize, cabbage and rice were downloaded from the TAIR (http://www.Arabidopsis.org/), gramene (http://ensembl. gramene.org/Zea_mays/Info/Index), BRAD (http:// brassicadb.org/brad/index.php) and RGAP (http://rice. plantbiology.msu.edu/) databases, respectively. Multiple alignments of the mature proteins were carried out and phylogenetic tree was built using MEGA 5.0, with the neighbour-joining (NJ) method and 1000 bootstrap replications. After that, the results were imported to the iTOL (https://itol.embl.de/) online service output picture.

\section{Protein motif and gene structure analysis}

The predicted barley and Qingke nsLTP protein sequences were submitted to the online MEME (http:// meme-suite.org/tools/meme) to identify 20 distinct conserved motifs in the nsLTPs. The following parameters were used: repetitions are arbitrary, maximum number of bases is 10 , and optimal base widths are limited to between 6 and 50 residues.

The prediction analysis of gene structure was carried out using GSDS (http://gsds.cbi.pku.edu.cn/) using the DNA and cDNA sequences of each predictive HvLTP and HtLTP gene from the barley and Qingke genomes.

\section{Chromosomal mapping and gene duplications}

The chromosome location information of the nsLTPs was searched in the barley and Qingke genome databases and MapInspect software was used to generate chromosomal distribution images.

Gene duplication was investigated following the method described by Kong et al [56]. The MCScanx and Circos programs were used to retrieve and map the collinearity between different plant genomes.

\section{Promoter analysis of HvLTPs and HtLTPs}

To investigate the cis-elements in promoter sequences of barley and Qingke nsLTP genes, the upstream sequences ( $1500 \mathrm{bp}$ ) of each identified HvLTP and HtLTP were retrieved from the barley and Qingke genomes using a Perl script. The PlantCARE website (http:// bioinformatics.psb.ugent.be/webtools/plantcare/ html/) was used to identify cis-elements in the promoters.

\section{Tissue expression profile analysis of HvLTPs}

The publicly available Barley RNA-Seq datasets were downloaded from the IPK database, and the value of fragments per kilobase of transcript per million fragments mapped (FPKM) of these genes was used visualize the heat map using HEMI software and then used to analyse the barley tissue expression profiles of the identified $H v L T P$ genes.

\section{RNA isolation and quantitative real-time PCR}

Total RNA was extracted using the Plant RNA isolation kit (Takara, Shiga-ken, Japan) following the manufacturer's instructions. The RNA quality was checked using a $1.0 \%(\mathrm{w} / \mathrm{v})$ agarose gel stained with ethidium bromide (EB), and the RNA samples were inspected for quality and quantity using a NanoDrop spectrophotometer and gel imager analysis. First-strand cDNA was synthesized from DNase-treated RNA with a PrimerScript 1st Strand cDNA synthesis kit (TIANGEN, Beijing, China). HvLTP and HtLTP gene-specific primers were designed based on their coding sequences (CDSs) using an online tool in NCBI (https: //www.ncbi.nlm.nih.gov/tools/primerblast) and then synthesized commercially (Shenggong, Shanghai, China) (Table S3). qRT-PCR was performed with SYBR GREENI and the CFX96 Real-time System (Bio-Rad, France) by strictly following the manufacturer's instructions. The thermal cycling conditions were as follows: $50^{\circ} \mathrm{C}$ for $2 \mathrm{~min}, 95^{\circ} \mathrm{C}$ for $10 \mathrm{~min}$, and $40 \mathrm{cy}$ cles of $95^{\circ} \mathrm{C}$ for $15 \mathrm{~s}$. The relative transcription levels were calculated using the $2^{-\Delta \Delta} \mathrm{CT}$ method, and three technical replicates were performed for each sample.

\section{Abbreviations \\ nsLTP: Plant non-specific lipid transfer proteins; $8 C M(E C M)$ : Eight cysteine residues motifs; IPK: Leibniz Institute of Plant Genetics and Crop Plant Research; NSS: N-terminal signal sequences; GPI: C-terminal glycosylphosphatidylinositol anchors; GRT-PCR: Quantitative real-time PCR}

\section{Supplementary Information}

The online version contains supplementary material available at https://doi org/10.1186/s12864-021-07958-8.

Additional file 1: Table S1. Query genes for the barley nsLTP gene family screening.

Additional file 2: Table S2. Gene duplication events for the HVLTP genes in each barley chromosome.

Additional file 3: Table S3. Quantitative PCR primers for the HVLTP (HtLTP) genes.

Additional file 4: Figure S1. Collinear analysis of the inter-species (A) and intra-species (B) comparison of HvLTP genes.

Additional file 5: Figure S2. Expression analysis of type 2, type $D$ and type G HvLTP genes.

\section{Acknowledgements}

Not applicable.

Authors' contributions

J.D., H.X. and R.D. conceived and designed the experiments. J.D., X.W. and H.X. performed the experiments and participated in the data analysis. Y.L. and C.Z. performed the qRT-PCR experiments. R.D. and J.S. revised the manuscript and improved the English. All authors reviewed and approved the final manuscript. 


\section{Funding}

This study was supported by the Applied Basic Research Project of Qinghai Science and Technology Department of China (2016-ZJ-772, 2019-ZJ-7049) and the National Natural Science Foundation of China (No. 31560052). The funding agencies did not have a role in the study design, the collection, analysis and interpretation of the data, or the writing of the manuscript.

\section{Availability of data and materials}

All data generated or analysed during this study are included in this article and its additional files. The genome data of Hordeum vulgare was downloaded from https://webblast.ipk-gatersleben.de/downloads/barley pangenome/Morex/ and http://plants.ensembl.org/Hordeum_vulgare/Info/ Index [29, 30], the genome data of Qingke were downloaded from https:// ftp.ncbi.nlm.nih.gov/genomes/genbank/plant/Hordeum_vulgare/all_ assembly_versions/GCA_004114815.1_Hulless_Barley_ass.V2/ and http:// www.ibgs.zju.edu.cn/ZJU_barleygenome.htm [22, 31], All the RNA-Seq data are available at IPK from https://apex.ipk-gatersleben.de/apex/f?p=284:57.

\section{Declarations}

\section{Ethics approval and consent to participate}

The plant materials (barley and Qingke) used in this article were obtained from the Academy of Agriculture and Forestry of Qinghai University. All plant materials were provided free of charge and maintained in accordance with international guidelines. This article did not contain any studies with human participants or animals and did not involve any endangered or protected species.

\section{Consent for publication}

Not applicable.

\section{Competing interests}

The authors declare that they have no competing interests.

\section{Author details}

${ }^{1}$ College of Eco-Environmental Engineering, Qinghai University, Xining 810016, Qinghai Province, China. ²Qinghai Qaidam Vocational \& Technical College, Delingha 817000, Qinghai Province, China. ${ }^{3}$ College of Agriculture and Animal Husbandry, Qinghai University, Xining 810016, Qinghai Province, China.

Received: 30 April 2021 Accepted: 29 August 2021

Published online: 20 September 2021

\section{References}

1. Boutrot F, Guirao A, Alary R, Joudrier P, Gautier MF. Wheat non-specific lipid transfer protein genes display a complex pattern of expression in developing seeds. Biochim Biophys Acta. 2005;1730(2):114-25.

2. Kader JC. Lipid-Transfer Proteins in Plants. Annu Rev Plant Physiol Plant Mol Biol. 1996:47:627-54

3. Jose-Estanyol M, Gomis-Ruth FX, Puigdomenech P. The eight-cysteine motif, a versatile structure in plant proteins. Plant Physiol Biochem. 2004;42(5):35565.

4. Boutrot F, Chantret N, Gautier MF. Genome-wide analysis of the rice and Arabidopsis non-specific lipid transfer protein (nsLtp) gene families and identification of wheat nsLtp genes by EST data mining. BMC Genomics. 2008;9:86.

5. Lascombe MB, Bakan B, Buhot N, Marion D, Blein JP, Larue V, et al. The structure of "defective in induced resistance" protein of Arabidopsis thaliana, DIR1, reveals a new type of lipid transfer protein. Protein Sci. 2008;17(9): 1522-30.

6. Nieuwland J, Feron R, Huisman BA, Fasolino A, Hilbers CW, Derksen J, et al. Lipid transfer proteins enhance cell wall extension in tobacco. Plant Cell. 2005;17(7):2009-19.

7. Liu F, Zhang X, Lu C, Zeng X, Li Y, Fu D, et al. Non-specific lipid transfer proteins in plants: presenting new advances and an integrated functional analysis. J Exp Bot. 2015;66(19):5663-81.

8. Salminen TA, Blomqvist K, Edqvist J. Lipid transfer proteins: classification, nomenclature, structure, and function. Planta. 2016;244(5):971-97.
9. Liu W, Huang D, Liu K, Hu S, Yu J, Gao G, et al. Discovery, identification and comparative analysis of non-specific lipid transfer protein (nsLtp) family in Solanaceae. Genomics Proteomics Bioinformatics. 2010;8(4):229-37.

10. van Ree R. Clinical importance of non-specific lipid transfer proteins as food allergens. Biochem Soc Trans. 2002;30(Pt 6):910-3.

11. Salcedo G, Sanchez-Monge R, Diaz-Perales A, Garcia-Casado G, Barber D. Plant non-specific lipid transfer proteins as food and pollen allergens. Clin Exp Allergy. 2004;34(9):1336-41.

12. Hincha DK. Cryoprotectin: a plant lipid-transfer protein homologue that stabilizes membranes during freezing. Philos Trans R Soc Lond B Biol Sci. 2002;357(1423):909-16.

13. White AJ, Alison Dunn M, Brown K, Hughes MA. Comparative analysis of genomic sequence and expression of a lipid transfer protein gene family in winter barley. J Exp Bot. 1994;45(12):1885-92.

14. Molina A, Garcia-Olmedo F. Enhanced tolerance to bacterial pathogens caused by the transgenic expression of barley lipid transfer protein LTP2. Plant J. 1997:12(3):669-75.

15. Gorjanović S, Sužnjević D, Beljanski M, Hranisavljević J. Barley lipid-transfer protein as heavy metal scavenger. Environ Chem Lett. 2004;2(3):113-6.

16. Jung HW, Kim KD, Hwang BK. Identification of pathogen-responsive regions in the promoter of a pepper lipid transfer protein gene (CALTPI) and the enhanced resistance of the CALTPI transgenic Arabidopsis against pathogen and environmental stresses. Planta. 2005;221(3):361-73.

17. Li J, Gao G, Xu K, Chen B, Yan G, Li F, et al. Genome-wide survey and expression analysis of the putative non-specific lipid transfer proteins in Brassica rapa L. PLoS One. 2014;9(1):e84556.

18. Safi H, Saibi W, Alaoui MM, Hmyene A, Masmoudi K, Hanin M, et al. A wheat lipid transfer protein (TdLTP4) promotes tolerance to abiotic and biotic stress in Arabidopsis thaliana. Plant Physiol Biochem. 2015;89:64-75.

19. Janska A, Hodek J, Svoboda P, Zamecnik J, Prasil IT, Vlasakova E, et al. The choice of reference gene set for assessing gene expression in barley (Hordeum vulgare L.) under low temperature and drought stress. Mol Gen Genomics. 2013;288(11):639-49.

20. Hua W, Zhu J, Shang Y, Wang J, Jia Q, Yang J. Identification of suitable reference genes for barley gene expression under abiotic stresses and hormonal treatments. Plant Mol Biol Report. 2015;33(4):1002-12.

21. Zeng X, Guo Y, Xu Q, Mascher M, Guo G, Li S, et al. Origin and evolution of qingke barley in Tibet. Nat Commun. 2018:9(1):5433.

22. Dai $F$, Wang $X$, Zhang $X Q$, Chen $Z$, Nevo $E$, Jin G, et al. Assembly and analysis of a qingke reference genome demonstrate its close genetic relation to modern cultivated barley. Plant Biotechnol J. 2018;16(3):760-70.

23. Zeng X, Yuan H, Dong X, Peng M, Jing X, Xu Q, et al. Genome-wide Dissection of Co-selected UV-B Responsive Pathways in the UV-B Adaptation of Qingke. Mol Plant. 2020;13(1):112-27.

24. Skriver K, Leah R, Muller-Uri F, Olsen FL, Mundy J. Structure and expression of the barley lipid transfer protein gene Ltp1. Plant Mol Biol. 1992;18(3):585-9.

25. Bakan B, Hamberg M, Larue V, Prange T, Marion D, Lascombe MB. The crystal structure of oxylipin-conjugated barley LTP1 highlights the unique plasticity of the hydrophobic cavity of these plant lipid-binding proteins. Biochem Biophys Res Commun. 2009;390(3):780-5

26. Molina A, Garcia-Olmedo F. Developmental and pathogen-induced expression of three barley genes encoding lipid transfer proteins. Plant J. 1993;4(6):983-91.

27. Dunn MA, Goddard NJ, Zhang L, Pearce RS, Hughes MA. Low-temperatureresponsive barley genes have different control mechanisms. Plant Mol Biol. 1994:24(6):879-88

28. Pearce RS, Houlston CE, Atherton KM, Rixon JE, Harrison P, Hughes MA, et al. Localization of expression of three cold-induced genes, blt101, blt4. 9, and blt14, in different tissues of the crown and developing leaves of coldacclimated cultivated barley. Plant Physiol. 1998;117(3):787-95.

29. International Barley Genome Sequencing C, Mayer KF, Waugh R, Brown JW, Schulman A, Langridge $P$, et al. A physical, genetic and functional sequence assembly of the barley genome. Nature. 2012;491(7426):711-6.

30. Mascher M, Gundlach H, Himmelbach A, Beier S, Twardziok SO, Wicker T, et al. A chromosome conformation capture ordered sequence of the barley genome. Nature. 2017;544(7651):427-33.

31. Zeng $X$, Long $H$, Wang $Z$, Zhao $S$, Tang $Y$, Huang $Z$, et al. The draft genome of Tibetan hulless barley reveals adaptive patterns to the high stressful Tibetan Plateau. Proc Natl Acad Sci U S A. 2015;112(4):1095-100.

32. Zeng $X, X u T$, Ling $Z$, Wang $Y$, Li $X, X u$ S, et al. An improved high-quality genome assembly and annotation of Tibetan hulless barley. Sci Data. 2020; 7(1):139. 
33. Li F, Fan K, Ma F, Yue E, Bibi N, Wang M, et al. Genomic Identification and Comparative Expansion Analysis of the Non-Specific Lipid Transfer Protein Gene Family in Gossypium. Sci Rep. 2016;6:38948.

34. D'Agostino N, Buonanno M, Ayoub J, Barone A, Monti SM, Rigano MM. Identification of non-specific Lipid Transfer Protein gene family members in Solanum lycopersicum and insights into the features of Sola I 3 protein. Sci Rep. 2019;9(1):1607.

35. Vignols F, Lund G, Pammi S, Tremousaygue D, Grellet F, Kader JC, et al. Characterization of a rice gene coding for a lipid transfer protein. Gene. 1994;142(2):265-70.

36. Nielsen KK, Nielsen JE, Madrid SM, Mikkelsen JD. New antifungal proteins from sugar beet (Beta vulgaris L.) showing homology to non-specific lipid transfer proteins. Plant Mol Biol. 1996;31(3):539-52.

37. Choi AM, Lee SB, Cho SH, Hwang I, Hur CG, Suh MC. Isolation and characterization of multiple abundant lipid transfer protein isoforms in developing sesame (Sesamum indicum L.) seeds. Plant Physiol Biochem. 2008:46(2):127-39.

38. Arondel W, Vergnolle C, Cantrel C, Kader J. Lipid transfer proteins are encoded by a small multigene family in Arabidopsis thaliana. Plant Sci. 2000;157(1):1-12.

39. Wei K, Zhong X. Non-specific lipid transfer proteins in maize. BMC Plant Biol. 2014;14:281.

40. Zhang M, Kim Y, Zong J, Lin H, Dievart A, Li H, et al. Genome-wide analysis of the barley non-specific lipid transfer protein gene family. Crop J. 2019; 7(1):65-76.

41. Edstam MM, Viitanen L, Salminen TA, Edqvist J. Evolutionary history of the non-specific lipid transfer proteins. Mol Plant. 2011;4(6):947-64.

42. Edstam MM, Blomqvist K, Eklof A, Wennergren U, Edqvist J. Coexpression patterns indicate that GPI-anchored non-specific lipid transfer proteins are involved in accumulation of cuticular wax, suberin and sporopollenin. Plant Mol Biol. 2013;83(6):625-49

43. Debono A, Yeats TH, Rose JK, Bird D, Jetter R, Kunst $L$, et al. Arabidopsis LTPG is a glycosylphosphatidylinositol-anchored lipid transfer protein required for export of lipids to the plant surface. Plant Cell. 2009;21(4):1230-8.

44. Guerche P, Tire C, De Sa FG, De Clercq A, Van Montagu M, Krebbers E. Differential Expression of the Arabidopsis $2 S$ Albumin Genes and the Effect of Increasing Gene Family Size. Plant Cell. 1990;2(5):469-78.

45. Guo L, Yang H, Zhang X, Yang S. Lipid transfer protein 3 as a target of MYB96 mediates freezing and drought stress in Arabidopsis. J Exp Bot. 2013; 64(6):1755-67.

46. Wang HW, Hwang SG, Karuppanapandian T, Liu A, Kim W, Jang CS. Insight into the molecular evolution of non-specific lipid transfer proteins via comparative analysis between rice and sorghum. DNA Res. 2012;19(2):17994.

47. Kouidri A, Whitford R, Suchecki R, Kalashyan E, Baumann U. Genome-wide identification and analysis of non-specific Lipid Transfer Proteins in hexaploid wheat. Sci Rep. 2018;8(1):17087.

48. Lauga B, Charbonnel-Campaa L, Combes D. Characterization of MZm3-3, a Zea mays tapetum-specific transcript. Plant Sci. 2000;157(1):65-75.

49. Flagel LE, Wendel JF. Gene duplication and evolutionary novelty in plants. New Phytol. 2009;183(3):557-64.

50. Moore RC, Purugganan MD. The evolutionary dynamics of plant duplicate genes. Curr Opin Plant Biol. 2005;8(2):122-8.

51. Fan K, Wang M, Miao Y, Ni M, Bibi N, Yuan S, et al. Molecular evolution and expansion analysis of the NAC transcription factor in Zea mays. PLoS One. 2014;9(11):e111837.

52. Federico ML, Kaeppler HF, Skadsen RW. The complex developmental expression of a novel stress-responsive barley Ltp gene is determined by a shortened promoter sequence. Plant Mol Biol. 2005;57(1):35-51.

53. Rius SP, Grotewold E, Casati P. Analysis of the P1 promoter in response to UV-B radiation in allelic variants of high-altitude maize. BMC Plant Biol. 2012; 12:92.

54. Xu XH, Huang XW, Qun L, Li YN, Wang Y, Liu C, et al. Two functional loci in the promoter of EPAS1 gene involved in high-altitude adaptation of Tibetans. Sci Rep. 2014:4:7465.

55. Zhang Z, Li J, Pan Y, Li J, Zhou L, Shi H, et al. Natural variation in CTB4a enhances rice adaptation to cold habitats. Nat Commun. 2017;8:14788.

56. Kong X, Lv W, Zhang D, Jiang S, Zhang S, Li D. Genome-wide identification and analysis of expression profiles of maize mitogen-activated protein kinase kinase kinase. PLoS One. 2013;8(2):e57714.

\section{Publisher's Note}

Springer Nature remains neutral with regard to jurisdictional claims in published maps and institutional affiliations.
Ready to submit your research? Choose BMC and benefit from:

- fast, convenient online submission

- thorough peer review by experienced researchers in your field

- rapid publication on acceptance

- support for research data, including large and complex data types

- gold Open Access which fosters wider collaboration and increased citations

- maximum visibility for your research: over $100 \mathrm{M}$ website views per year

At BMC, research is always in progress.

Learn more biomedcentral.com/submissions 